\title{
R-matrix study for electron scattering of beryllium dihydride for fusion plasma
}

Dhanoj Gupta ${ }^{1}$, Mi-Young Song ${ }^{1 *}$, Heechol Choi ${ }^{1}$, Deuk-Chul Kwon ${ }^{1}$, K. L. Baluja ${ }^{2}$ and Jonathan Tennyson ${ }^{3}$

${ }^{1}$ Plasma Technology ResearchCenter, National Fusion Research Institute, 37

Dongjangsan- ro, Gunsan, Jeollabuk-do 54004, South Korea.

(Formerly) ${ }^{2}$ Department of Physics \& Astrophysics, University of Delhi, Delhi110007, India

${ }^{3}$ Department of Physics and Astronomy, University College London, Gower St., London WCIE 6BT, United Kingdom.

Email:mysong@nfri.re.kr

\begin{abstract}
We report the integral elastic, differential, momentum transfer, dissociative electron attachment and electronic and rotational excitation cross sections for the lowenergy electron impact on beryllium dihydride $\left(\mathrm{BeH}_{2}\right)$ computed using the Quantemol$\mathrm{N}$ interface for driving the UK molecular R-matrix code. The energy of the projectile electron is in the range $0.1-10 \mathrm{eV}$. The effect of multichannel coupling is investigated by calculating the cross sections with various target models by increasing the number of target states in the trial wavefunction of the entire scattering system. The cross sections converge for the calculation with more than 15-target states. The vertical excitation energies calculated with the present model give excellent agreement with the EOM-CCSD calculations. Collisional frequencies are determined using the momentum transfer cross section for a Maxwell-Boltzmann distribution. From the collisional frequency, the transport properties such as mean free path, diffusivity and mobility are calculated for the temperature range of 100 to $500000 \mathrm{~K}$. The cross section data and transport properties reported in this article are important for fusion plasma.
\end{abstract}

\section{Introduction}

Electron collision with atoms, ions, molecules and surfaces are of fundamental importance in low temperature plasmas (LTPs) with applications to wide variety of plasma using technologies [1]. The motivation of the present article is to undertake an ab-initio study of electron collision cross section of $\mathrm{BeH}_{2}$ within the complete active space configuration interaction (CAS-CI) and static exchange (SE) models using the UK molecular R-matrix method. Cross sections for elastic and inelastic processes such as elastic, differential, momentum transfer, dissociative electron attachment (DEA) and excitation cross section are calculated for energies below 10 $\mathrm{eV}$. The role of multichannel effect on the various cross sections is studied at low energies. The study of electron molecule collision at low impact energies remains a computational challenge, which is evident from the fact that to the best of our knowledge there are no studies of electron collision with the simple $\mathrm{BeH}_{2}$ molecule.

$\mathrm{BeH}_{2}$ is particularly important for the fusion plasma applications. In the International thermonuclear experimental reactor (ITER), currently under construction, beryllium is planned to be used as one of the wall materials and hence will be directly exposed to plasma 
environment in the ITER [2]. The interaction of plasma containing hydrogen and is its isotope in the edge and divertor region with the Be walls will lead to the formation of Be hydrides such as $\mathrm{BeH}$ and $\mathrm{BeH}_{2}[3,4]$. Due to the low temperature $(\sim 0.5-100 \mathrm{eV})$ in the edge and divertor plasma, various collisional processes and reactions could be triggered between these species and the main plasma constituents $\left(\mathrm{e}, \mathrm{H}^{+}, \mathrm{H}, \mathrm{H}_{2}\right.$ and $\left.\mathrm{H}_{2}{ }^{+}\right)$in these regions [5].The interaction of free electrons in the divertor and edge plasma could have a substantial effect on the plasma itself. Hence, the study of various electron collision processes (elastic and inelastic cross sections) with the species present in such environments is important for the study of beryllium transport in the plasma and influxes of beryllium hydrides into the plasma due to the plasmaberyllium wall interactions [5]. BeH (BeD) emission spectra have been observed in JET (the Joint European Taurus) [6,7], which is currently run with Be-coated walls. At present the status of $\mathrm{BeH}_{2}\left(\mathrm{BeD}_{2}\right)$ in the JET divertor region remains a matter for speculation.

There is a lack of cross section data for the important $\mathrm{BeH}_{2}$ molecule. There is just one study of the total electron-impact ionization cross section by Maihom et al. [8] using the binary encounter-Bethe (BEB) method. While, the electron collision processes such as elastic and inelastic, have been extensively studied for $\mathrm{BeH}$ using the R-matrix method [5,9], we are not aware of any electron collision studies with $\mathrm{BeH}_{2}$ other than for ionization. Hence, in the present article we compute elastic, differential cross section (DCS), momentum transfer cross section (MTCS), dissociative electron attachment (DEA), electronic and rotational excitation cross sections at energies relevant for studies of fusion plasmas. These calculations are augmented by studies of collisional frequencies and the transport properties such as mean free path, diffusivity and mobility, which are calculated assuming a Maxwell-Boltzmann distribution.

The present article is organised in the following manner. The next section describe the target models used for the low energy cross section calculations. In section III the molecular R-matrix method is described briefly, while in section IV the graphical representation of the results are presented with discussions and section V summarises the outcome of the present study.

\section{Target models used for the present calculation}

The $\mathrm{BeH}_{2}$ molecule is a linear, closed shell system belonging to the $D_{\infty h}$ natural point group symmetry. However, none of the codes used in the present study can work with this point group, so all calculations reported below used $C_{2 v}$ point group symmetry. The experimental molecular geometry of this target was obtained from the Computational Chemistry Comparison and Benchmark Database (CCCBDB) [8] website which gives the equilibrium bond length of Be$\mathrm{H}$ as $1.326 \AA$. A Hartree-Fock (HF) self-consistent field (SCF) calculation with a $6-31 \mathrm{G}^{*}$ Gaussian-type orbital (GTO) basis set was used to generate a wave function for the ground state of $\mathrm{BeH}_{2}$ molecule. The electronic configuration of the ground state of $\mathrm{BeH}_{2}$ is $1 \mathrm{a}_{1}^{2}, 2 \mathrm{a}_{1}{ }^{2}$, $3 \mathrm{a}_{1}{ }^{2}\left(1 \sigma_{\mathrm{g}}{ }^{2}, 2 \sigma_{\mathrm{g}}{ }^{2}, 3 \sigma_{\mathrm{g}}{ }^{2}\right)$ in $C_{2 v}\left(D_{\infty}\right)$ symmetry. The $\mathrm{BeH}_{2}$ target states were represented using the complete active space (CAS) configuration interaction (CI) method. In our CAS-CI model, two electrons were frozen in the core $1 \sigma_{\mathrm{g}}$ molecular orbital (MO). Previous studies on BeH suggest that correlation of these electrons can safely be neglected [9]. The remaining four electrons are allowed to move freely in the nine MOs $\left(2 a_{1}-6 a_{1}, 1 b_{1}-2 b_{1}, 1 b_{2}-2 b_{2}\right)$. The maximum number of target states included in the present calculation is 25 , the number of configuration 
state functions (CSFs) generated for the ground state is 172, and the number of scattering channels are 141. The absolute value of the ground state energy obtained in the 25 -state calculation is -15.78 Hartree. By symmetry, the dipole moment of the molecule is zero and is evident from the present calculations. The vertical excitation energies (VEE) of all the twentyfive excited states obtained from the present CAS-CI model are given in Table 1. These energies are compared with an EOM-CCSD (equation of motion - coupled clusters singles and doubles) results calculated using the Gaussian 09 [11] suite of program using the larger ccpVTZ basis set as part of the present study.

Table 1: $\mathrm{BeH}_{2}$ vertical excitation energy computed with the CAS-CI and EOM-CCSD methods; also show are the symmetry-allowed CAS-CI dipole transition moments (and permanent quadrupole moments).

\begin{tabular}{|c|c|c|c|c|}
\hline $\begin{array}{l}\text { State } \\
\left(\mathbf{C}_{2 \mathrm{v}}\right)\end{array}$ & $\begin{array}{l}\text { State } \\
\left(\mathbf{D}_{\infty h}\right)\end{array}$ & $\begin{array}{c}\text { Energy (eV) } \\
\text { (CAS-CI) }\end{array}$ & $\begin{array}{l}\text { Energy (eV) } \\
\text { EOM-CCSD }\end{array}$ & Transition moments (au) \\
\hline${ }^{1} \mathrm{~A}_{1}$ & ${ }^{1} \Sigma_{\mathrm{g}}^{+}$ & 0.0 & 0.0 & $0.0,(3.4541)$ \\
\hline${ }^{3} \mathrm{~B}_{1} /{ }^{3} \mathrm{~B}_{2}$ & ${ }^{3} \Pi_{\mathrm{g}}$ & 6.15 & 6.147 & $(-3.1708,3.3643)$ \\
\hline${ }^{1} \mathrm{~B}_{1} /{ }^{1} \mathrm{~B}_{2}$ & ${ }^{1} \Pi_{g}$ & 6.48 & 6.548 & $0.0,(-3.5048,3.4926)$ \\
\hline${ }^{3} \mathrm{~B}_{1} /{ }^{3} \mathrm{~B}_{2}$ & ${ }^{3} \Pi_{u}$ & 7.19 & 7.046 & $(-2.1279,3.3201)$ \\
\hline${ }^{3} \mathrm{~A}_{1}$ & ${ }^{3} \Sigma_{\mathrm{u}}^{+}$ & 8.79 & 8.588 & $(-1.7107)$ \\
\hline${ }^{1} \mathrm{~B}_{1} /{ }^{1} \mathrm{~B}_{2}$ & ${ }^{1} \Pi_{u}$ & 9.08 & 8.717 & $-1.4674,(-0.0927,3.7184)$ \\
\hline${ }^{3} \mathrm{~A}_{1}$ & ${ }^{3} \sum_{\mathrm{g}}{ }^{+}$ & 10.71 & 9.744 & $(0.0872)$ \\
\hline${ }^{1} \mathrm{~A}_{1}$ & ${ }^{1} \Sigma_{\mathrm{g}}^{+}$ & 11.14 & - & $(-1.8157)$ \\
\hline${ }^{1} \mathrm{~A}_{1}$ & ${ }^{1} \Sigma_{\mathrm{u}}^{+}$ & 11.36 & - & $1.3713,(0.0267)$ \\
\hline${ }^{3} \mathrm{~A}_{2}$ & ${ }^{3} \Sigma_{\mathrm{g}}^{-}$ & 12.23 & - & $(-7.3653)$ \\
\hline${ }^{3} \mathrm{~A}_{1}$ & ${ }^{3} \Sigma_{\mathrm{u}}^{+}$ & 12.79 & - & $(2.5032)$ \\
\hline${ }^{3} \mathrm{~A}_{2}$ & ${ }^{3} \Sigma_{\mathrm{u}}^{-}$ & 13.27 & - & $(-7.1653)$ \\
\hline${ }^{1} \mathrm{~A}_{2} /{ }^{1} \mathrm{~A}_{1}$ & ${ }^{1} \Delta_{\mathrm{g}}$ & 13.30 & - & $(-7.4110)$ \\
\hline${ }^{3} \mathrm{~A}_{2} /{ }^{3} \mathrm{~A}_{1}$ & ${ }^{3} \Delta_{\mathrm{u}}$ & 13.32 & - & $(-7.3718)$ \\
\hline${ }^{3} \mathrm{~A}_{1}$ & ${ }^{3} \Sigma^{+}{ }_{\mathrm{u}}$ & 13.40 & - & $(0.0600)$ \\
\hline${ }^{1} \mathrm{~A}_{1}$ & ${ }^{1} \Sigma_{\mathrm{g}}^{+}$ & 13.75 & - & $(0.0573)$ \\
\hline${ }^{1} \mathrm{~A}_{2}$ & ${ }^{1} \Sigma_{\mathrm{u}}^{-}$ & 14.41 & - & $(-6.6582)$ \\
\hline${ }^{3} \mathrm{~A}_{1}$ & ${ }^{3} \Sigma_{\mathrm{u}}^{+}$ & 14.49 & - & $(-3.7782)$ \\
\hline${ }^{1} \mathrm{~A}_{1}$ & ${ }^{1} \Sigma_{\mathrm{u}}^{+}$ & 14.59 & - & $0.0876,(3.0011)$ \\
\hline
\end{tabular}

The degeneracy structure and selection rules for dipole transitions can be used to give full $D_{\infty} h$ state designations. The present CAS-CI target model gives excellent agreement of the VEE with the VEE calculated using the EOM-CCSD method for the first six excited states in the present study. The first three excited are the $\Pi$ states in the natural $D_{\infty}$ point group symmetry. The threshold for the first excited state in our CAS-CI model is $6.15 \mathrm{eV}$, which is in excellent agreement with the first excitation threshold at 6.147, eV calculated using the EOM-CCSD method. The comparison for higher excited states are also in good agreement with the EOMCCSD method as shown in Table 1. The good comparison of the target properties suggest that our target model is reliable for the scattering studies. 
Since our calculations only considered a linear symmetric molecule, the dipole moment of each state is identically zero. Table 1 also shows the transition moments from the ground state to dipole allowed transitions, which is the first entry under the heading of transition moments. We have calculated the electronic transition moments from the ground ${ }^{1} \Sigma^{+}$g state to the lowest excited singlet spin states, ${ }^{1} \Pi_{\mathrm{u}}$ and ${ }^{1} \Sigma^{+}{ }_{\mathrm{u}}$, which have large values -1.4674 au and $1.3713 \mathrm{au}$, respectively. For triplet states, these dipole transitions are zero because these are spin forbidden. The quadrupole moments of each state is also shown within the parenthesis, the first entry refers to the component $\mathrm{Q}_{20}$ whereas the second entry refers to $\mathrm{Q}_{22}$. For molecules, the $\mathrm{Q}_{20}$ moment represents the parallel component which is proportional to the expectation value of the operator $\left[z^{2}-\left(x^{2}+y^{2}\right) / 2\right]$ whereas the perpendicular component $\mathrm{Q}_{22}$ represents the expectation value $\left[\left(\mathrm{x}^{2}-\mathrm{y}^{2}\right) / 2\right]$. The difference between these two components is a measure of the anisotropy of the particular excited state. This anisotropy is maximum for the lowest ${ }^{1} \Pi_{u}$ excited state. For $\Sigma$ states there is only parallel component $\mathrm{Q}_{20}$.

\section{Theoretical methodology: R-matrix method}

There are a number of computational procedures for treating low-energy electron-molecule collisions; here we employ the R-matrix method [12, 13]; however, the Schwinger multichannel (SMC) [14] and Kohn variation [15] methods are also widely used for low energy calculations. All these methods are found to predict cross sections for variety of targets successfully over the years and indeed to give similar results when using the same target and scattering model. The R-matrix method is probably the most commonly used ab-initio method for low-energy electron atom/molecule collision calculations.

Our calculations used the UK polyatomic R-matrix codes [16] as run using the Quantemol-N expert system [17]. The calculations are performed in a fixed nuclei (FN) approximation. The basic idea behind the R-matrix method is in the division of the configuration space into an inner and outer region. The wave function of the target under study must be contained inside the inner region, where the center-of-mass of the target coincides with the origin of the coordinates. Due to the strong interaction of the scattering electron and the target inside the inner region, we need to consider the various interactions such as static, exchange, polarization and correlation between the target and the projectile. Appropriately adapted quantum chemistry codes are used for the inner region solution [13]. All the target properties are calculated in this region. The beauty of the R-matrix method lies in the fact that we need to solve the inner region problem only once for each given total scattering symmetry, independent of the electron collision energy, which makes this method computational viable and easy.

Initially, the wave function for the $(N+1)$ electron system is constructed inside the inner region, which is given by a close-coupling (CC) [18] approximation,

$$
\psi_{k}^{N+1}=A \sum_{i j} a_{i j k} \Phi_{i}^{N}\left(x_{1}, \ldots, x_{N}\right) u_{i j}\left(x_{N+1}\right)+\sum_{i} b_{i k} \chi_{i}^{N+1}\left(x_{1}, \ldots, x_{N+1}\right)
$$

Here the first summation runs over the target plus continuum states used in the close-coupled expansion. $A$ is an anti-symmetrization operator, which is needed to allow for the exchange between the scattering and target electrons, $\Phi_{i}^{N}$ is the wave function of the $i$ th target state and $x_{N}$ is the spatial and spin coordinate of the $N$ th target electron. $u_{i j}$ are the continuum orbitals 
used to represent the scattering electron. $a_{i j k}$ and $b_{i k}$ are variational coefficients determined as a results of the diagonalization of Hamiltonian matrix which is constructed using an especially adapted procedure [19]. Short-range correlations and polarization effects are taken care by the second summation, where the $\chi_{i}^{N+1}$ are called $L^{2}$ configurations. These $\chi_{i}$ are multi-centre quadratically integrable functions constructed by placing all the $N+1$ electrons in target MOs and are important for balance of the calculation. However, their inclusion leads to the appearance of a pseudo-resonant structure in cross sections of electron molecule collisions at higher collision energies, as well as to a significant increase in the number of integrodifferential equations to be solved. However the B-spline R-matrix method [20-23] resolve many of these problems and, in particular allows the use much larger R-matrix radii so that the inner region can incorporate diffuse functions to represent Rydberg electronic states without the problem of linear dependence. A molecular R-matrix code based on B-splines is in advanced stages of development, see Ref. [9].

A set of MOs were constructed from occupied and virtual target MOs from the HF-SCF calculation augmented with GTO continuum orbitals from Faure et al. [24]. The calculation was performed with the continuum orbitals up to $g$ partial waves. Modelling of correlation/polarization effects can be improved by increasing the number of target states in the calculations. However, once the convergence of the results is obtained, increasing the number of target states further has minimal effect on the cross sections.

Once the inner region problem is solved, the solution is propagated to the boundary where the R-matrix acts as a bridge and connects the inner region solution to the outer region. In the present calculation, the inner region is defined by sphere of radius $10 \mathrm{a}_{0}$. The R-matrix is propagated outwards up to 100 a 0 to the asymptotic region where it is matched with asymptotic functions obtained from a Gailitis expansion [25]. The outer region problem is comparatively easy compared to the inner region as the scattering electron is far from the molecular charge cloud and hence exchange and correlation effects are neglected. In this region, we need to consider only the long-range multipolar interactions between the scattering electron and the target. However, the energy dependence is explicitly treated in the outer region. The coupled single-center equations describing the scattering are integrated to give the asymptotic $K$-matrix, which is subsequently used to deduce all the observables in this region such as cross sections, eigenphase and resonances via the $T$-matrices defined as follows,

$$
T=\frac{2 i K}{1-i K}
$$

Moreover, the $K$-matrices were further employed in the POLYDCS program of Sanna and Gianturco [26] for the evaluation of the differential cross sections (DCS) and momentum transfer cross sections (MTCS). One of the important characteristics of the low energy cross sections are the resonances. In the present study, the resonance detection program RESON [27] was used to detect resonances and width by fitting it to the Breit-Wigner profile [28]. 


\section{Results and discussions}

In the section the graphical representation of the results obtained in the present study are presented along with the discussions. The results are plotted in Figs. 1-8. Table 2 gives the resonance position and width obtained at low energies for electron scattering from $\mathrm{BeH}_{2}$

Table 2: Resonance position and width of e-BeH2 scattering for a 25-state CC calculations

\begin{tabular}{|c|c|c|}
\hline \multirow{2}{*}{ State } & \multicolumn{2}{|c|}{ Present } \\
\cline { 2 - 3 } & Position (eV) & Width (eV) \\
\hline${ }^{2} \Pi_{\mathrm{u}}\left({ }^{2} \mathrm{~B}_{1}{ }^{2} \mathrm{~B}_{2}\right)$ & 0.45 & 0.40 \\
\hline${ }^{2} \Delta_{\mathrm{g}}\left({ }^{2} \mathrm{~A}_{1} /{ }^{2} \mathrm{~A}_{2}\right)$ & 6.24 & 0.02 \\
\hline${ }^{2} \Sigma_{\mathrm{g}}{ }^{-}\left({ }^{2} \mathrm{~A}_{2}\right)$ & 7.37 & 0.40 \\
\hline${ }^{2} \Sigma_{\mathrm{g}}{ }^{+}\left({ }^{2} \mathrm{~A}_{1}\right)$ & 7.44 & 0.38 \\
\hline
\end{tabular}

\subsection{Elastic cross section}
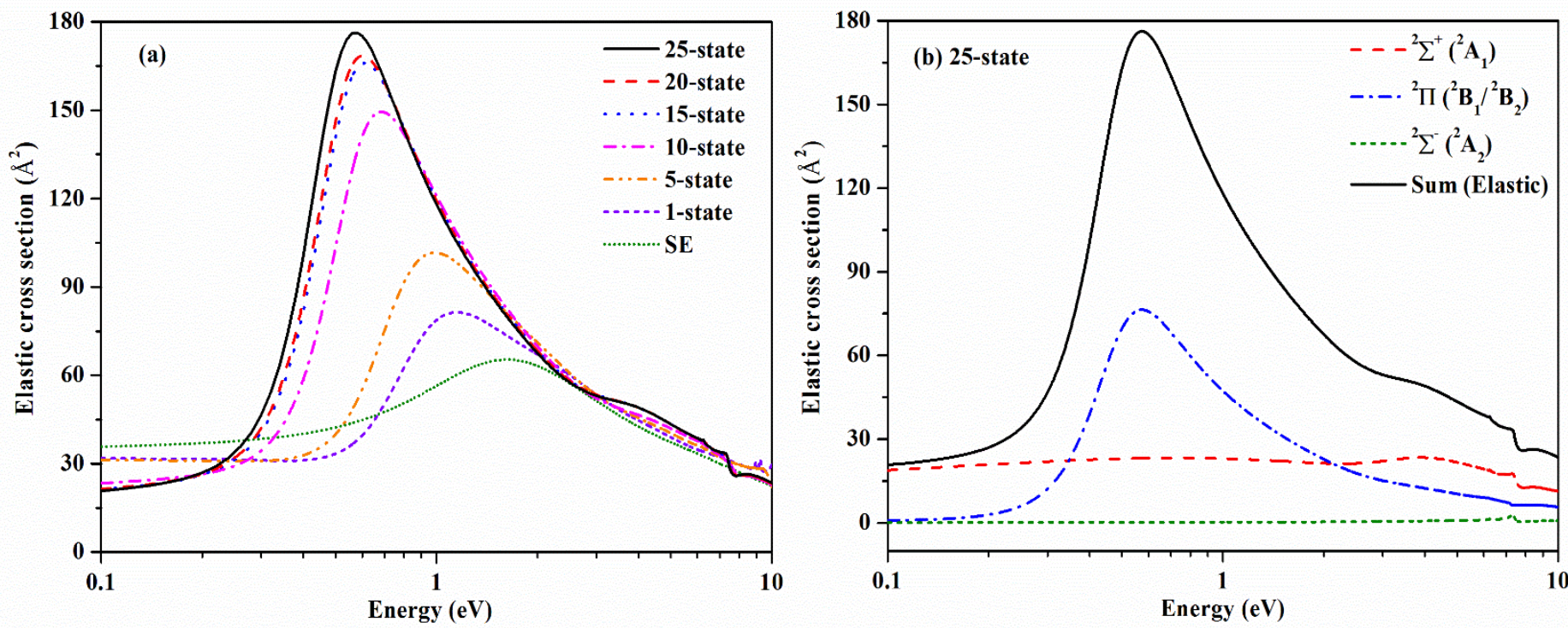

Figure 1. Elastic cross section for (a) SE, 1-state, 5-state, 10-state, 15-state, 20-state and 25state $\mathrm{CC}$ calculations in the energy range of 0.1 to $10 \mathrm{eV}$ (b) the doublet symmetry components ${ }^{2} \mathrm{~A}_{1},{ }^{2} \mathrm{~B}_{1} /{ }^{2} \mathrm{~B}_{2}$ and ${ }^{2} \mathrm{~A}_{2}$ along with summed elastic cross sections for the 25 -state model.

Figure 1(a) shows elastic cross section for SE, 1-state, 5-state, 10-state, 15-state, 20-state and 25 -state $\mathrm{CC}$ calculations in the energy range from 0.1 to $10 \mathrm{eV}$. A broad peak is observed at around $1.5 \mathrm{eV}$ in the $\mathrm{SE}$ calculations, which subsequently shifts to the lower energies with the multichannel coupling in a 1-state, 5-state, 10-state, 15-state, 20-state and 25-state CC calculations. The magnitude of the elastic cross section also increases with the increase of the number of target states. As the number of target states increase there is enhancement of the polarization-correlation effects that provide additional attraction that pulls the resonance position towards lower energy and increases the peak value of the cross section. The cross section results almost converge for the 15,20 and 25 state calculations and we have found that 
the further increase of the target state giving the similar cross section as that of 25 -state CC calculation. The first broad peak around $0.45 \mathrm{eV}$ with a width of $0.40 \mathrm{eV}$ in a 25-state calculation is a ${ }^{2} \Pi_{u}$ shape resonance, which is characterized by a sharp change in the cross sections as seen in figure 1 (a).

Figure 1(b) shows the elastic cross sections of the four doublet components for 25 -state CC calculations, together with the summed elastic cross section. The ${ }^{2} \mathrm{~B}_{1}{ }^{2} \mathrm{~B}_{2}$ states are degenerate and are shown as a single curve in the figure. These two components contribute maximum to the elastic cross section and the shape resonance at $0.45 \mathrm{eV}$ in the elastic cross section is also due to these degenerate symmetry components, which can be seen as a broad peak in the ${ }^{2} \mathrm{~B}_{1} /{ }^{2} \mathrm{~B}_{2}$ cross section. The shape resonance is basically an anion state of $\mathrm{BeH}_{2}$ consisting of the ground state of the molecule plus an electron temporarily captured in the vacant $1 \pi_{\mathrm{u}} \mathrm{MO}$ forming the compound state ${ }^{2} \Pi_{u}$.

As shown in Table 2, we find a set of three resonances associated with the low-lying excited target states which generally have the configuration $1 \sigma_{\mathrm{g}}{ }^{2}, 2 \sigma_{\mathrm{g}}{ }^{2}, 3 \sigma_{\mathrm{g}}{ }^{1}, 1 \pi_{\mathrm{u}}{ }^{1}$. These narrower core excited shape resonances all have the configuration $1 \sigma_{\mathrm{g}}{ }^{2}, 2 \sigma_{\mathrm{g}}{ }^{2}, 3 \sigma_{\mathrm{g}}{ }^{1}, 1 \pi_{\mathrm{u}}{ }^{2}$; as discussed below, they play an important role in DEA for this system as the ${ }^{2} \Pi_{u}$ shape resonance is too low in energy for this channel to be open. It is also worth noting that there will be an equivalent set of states with quartet spins arising from the same configuration, but these states cannot be reached by electron collision with the singlet ground state.

\subsection{Differential and momentum transfer cross sections}

Figure 2 shows the DCS calculated for the energies 1, 3, 5, 7 and $10 \mathrm{eV}$ with DCS plotted along $\mathrm{y}$-axis in logarithmic scale and scattering angle in x-axis in a linear scale. In figure 2 (a)-2(e) the DCS for the five different CC models with 5, 10, 15, 20 and 25 target states are plotted together to investigate the influence of multichannel effects in the DCS. Models with 15, 20 and 25 states give similar cross sections for all the energies considered. This suggests that good convergence has been achieved with respect to the increasing multichannel effects. The multichannel effects are quite noticeable for all the energies, where the DCS show some variation for the 5 and 10 state calculations compared to 15,20 and 25 states CC calculations.

In figure 2 (f), the DCS for all the energies, 1, 3, 5, 7 and $10 \mathrm{eV}$ are plotted together to get an idea about the shape and nature of the cross section for the 25-state CC calculations. The DCS are obtained by summing up all the rotational transitions DCS involving $\Delta J$ up to 5 for each incident energy. The DCS decreases smoothly for each energies at the forward angle and shows a minima for 1 and $3 \mathrm{eV}$ at 100-110 degrees, while for other energies 5, 7 and $10 \mathrm{eV}$ it shows a minima at around 90-100 degrees. The DCS slowly increases in the backward direction after the minima for all the energies. The backward scattering is responsible for the enhancement of momentum transfer cross sections. 

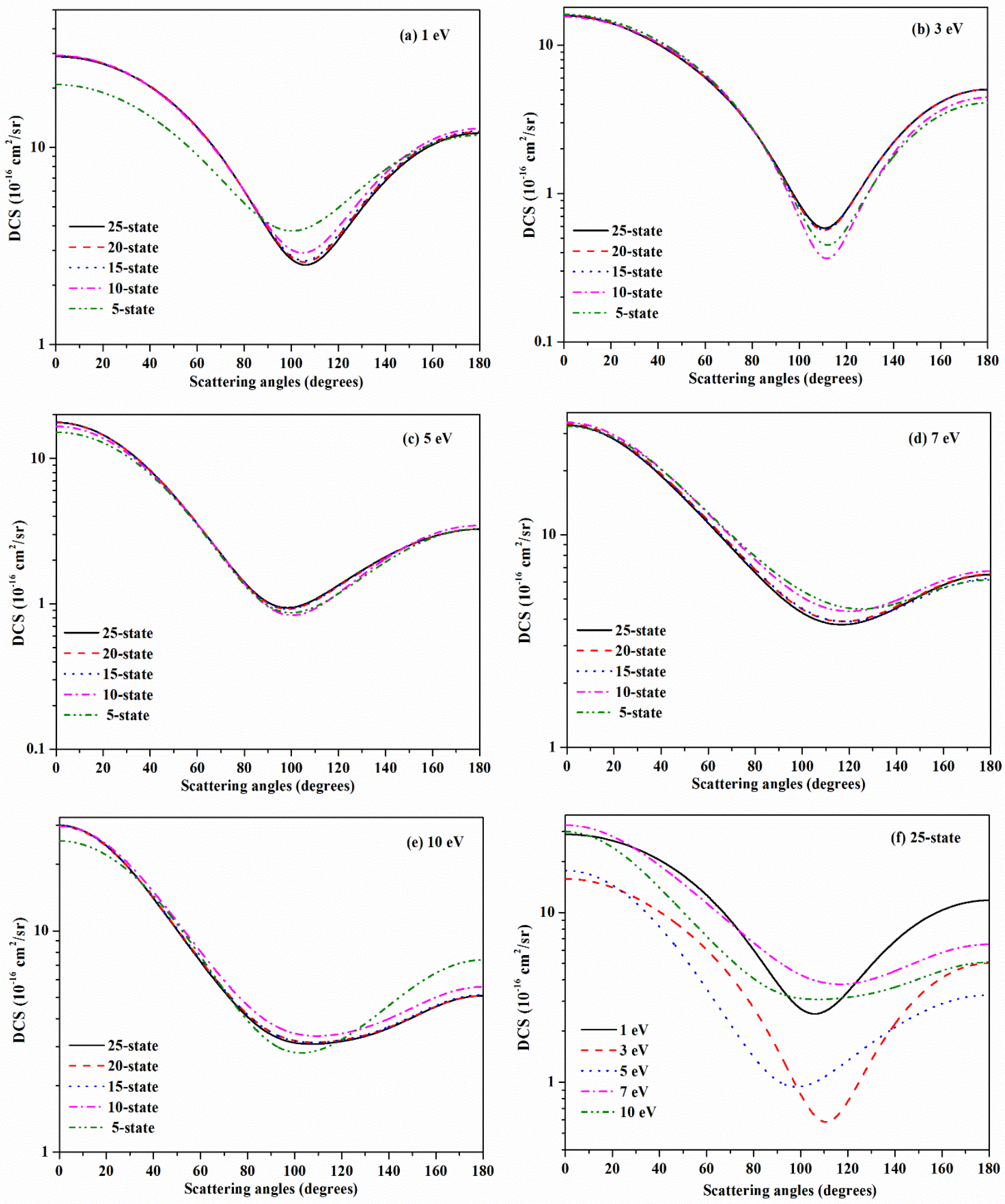

Figure 2. Differential cross section for elastic scattering at incident energies of 1, 3, 5, 7 and $10 \mathrm{eV}$ : (a)-(e) with five different models: 5-state, 10-state, 15-state, 20-state and 25-state CC calculations; (f) plotted together for 25-state CC calculations. 


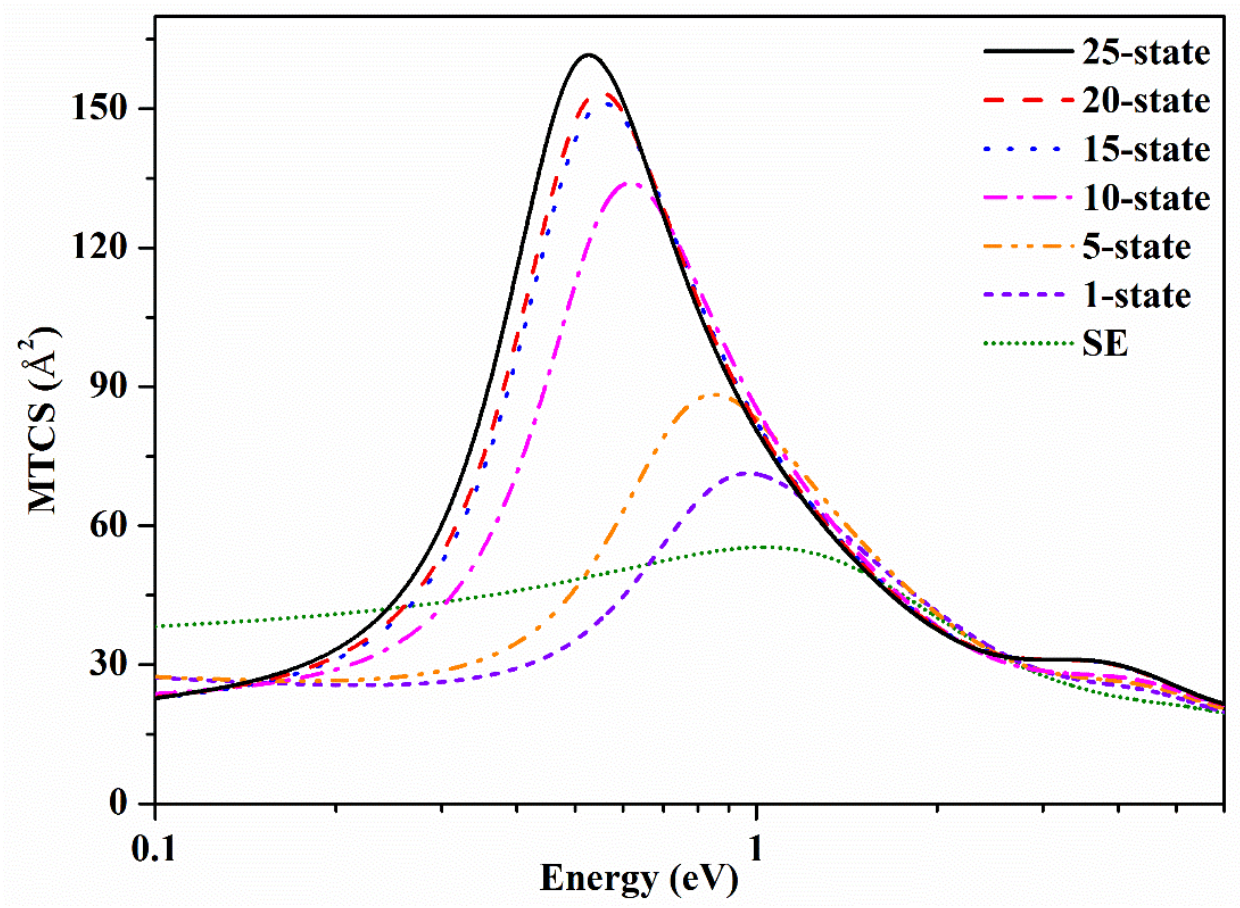

Figure 3. Momentum transfer cross section for SE, 1-state, 5-state, 10-state, 15-state, 20-state and 25-state CC calculations

The MTCS for the SE, 1-state, 5-state, 10-state, 15-state, 20-state and 25 state CC calculations are plotted in Fig. 3. The MTCS are calculated from the data of the DCS using the same program POLYDCS used for calculating DCS. Similar to the elastic cross section, the MTCS shows a broad peak at around $0.45 \mathrm{eV}$. This peak is characterized as the ${ }^{2} \Pi_{\mathrm{u}}$ shape resonance. It is also noticeable that the peak of the cross sections shifts to the lower energies with increasing number of target states. The results for 15, 20 and 25 state CC calculation almost give similar cross section data and the further increase of the target state gives similar results with that of 25 -state calculations.

\subsection{Electronic excitation cross section}

The electronic excitation cross sections are plotted for the first five excited state in a 25-state $\mathrm{CC}$ calculation in figure 4 (a). The threshold for the first excited state is at $6.15 \mathrm{eV}$ for the ${ }^{3} \Pi_{\mathrm{g}}$ state and for the second excited state, it is at $6.48 \mathrm{eV}$ for the ${ }^{1} \Pi_{\mathrm{g}}$ state. The threshold for the third excited state, ${ }^{3} \Pi_{\mathrm{u}}$ is at $7.19 \mathrm{eV}$. Excitation to the triplet excited states contribute most to the total cross section compared to other electronic excited states. There are peaks in the cross section near thresholds for $\Pi$ states due to p-partial wave threshold effect. These narrow peaks in the excitation cross section are associated with the resonances of core-excited character. These resonances are centred at 6.24, 7.37 and $7.44 \mathrm{eV}$ with corresponding scattering states ${ }^{2} \Delta_{\mathrm{g}},{ }^{2} \Sigma_{\mathrm{g}}{ }^{-}$and ${ }^{2} \Sigma_{\mathrm{g}}{ }^{+}$. All the core excited shape resonances have the configuration, $1 \sigma_{\mathrm{g}}{ }^{2} 2 \sigma_{\mathrm{g}}{ }^{2} 3 \sigma_{\mathrm{g}}{ }^{1}$ $1 \pi_{\mathrm{u}}^{2}$ and are listed in Table 2 .

Figures 4(b)-4(d) shows the excitation cross section from the ground state to the first three excited state in a 10-state, 15-state, 20-state and 25-state CC calculations. Except for the 10state calculation, all other calculations give similar cross section for these excited states 
showing that a good convergence is obtained for the excitation cross sections. Moreover the agreement of the vertical excitation energies with that of EOM-CCSD calculations suggest that the uncertainty in the cross section is small. The sensitivity of the cross section is also checked by performing the calculations using different basis sets and also by increasing the radius of the inner region. The deviation in the excitation cross section calculated using different models is quite small suggesting that the uncertainty in the cross section is less mostly within $10 \%$.
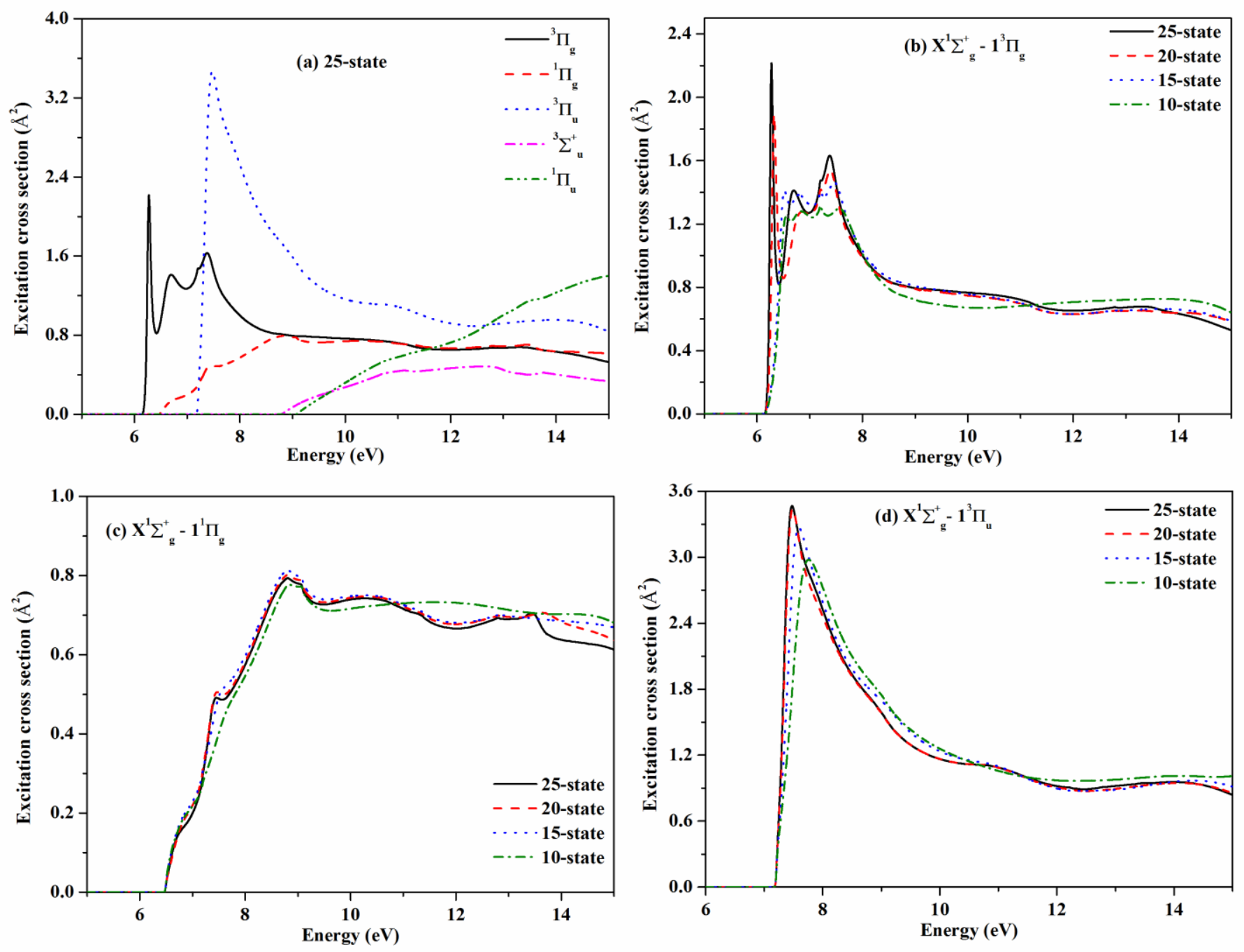

Figure 4. Electronic excitation cross section for the (a) first five excited states plotted together in 25-state CC calculations; (b)-(d) first three excited states with a 10-state, 15-state, 20-state and 25-state CC calculations.

\subsection{Rotational excitation cross sections}

$\mathrm{BeH}_{2}$ is a symmetric molecule which does not possess a permanent dipole moment so rotational excitation only involves transitions with $\Delta J$ even. Rotational cross section for the allowed transition starting from $J=0$ are depicted in figure 5 for energies up to $6 \mathrm{eV}$. Cross sections for the transition $J=0 \rightarrow 0,0 \rightarrow 2$ show structure at the energy of the shape resonance detected in 
the present study. The magnitude of the cross section for the rotationally elastic $J=0 \rightarrow 0$ transition is much larger than the $J=0 \rightarrow 2,0 \rightarrow 4$ transitions; this behaviour is usual for systems with no permanent dipole moment.

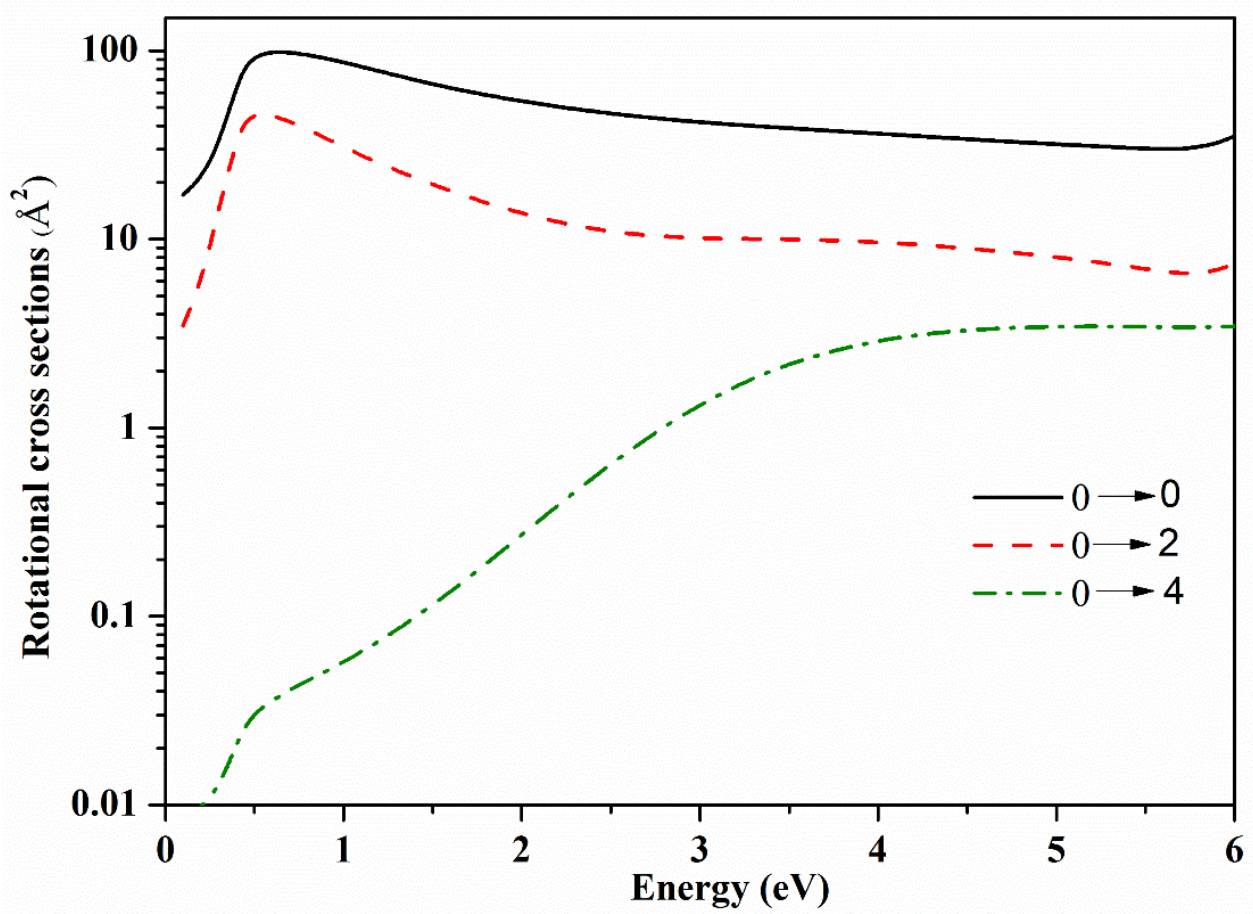

Figure 5. Rotational cross section of $\mathrm{BeH}_{2}$ in its ground state for the $J=0 \rightarrow 0,0 \rightarrow 2,0 \rightarrow 4$ transition.

4.5. Dissociative electron attachment cross section of $\mathrm{H}^{-}$fragment of $\mathrm{BeH}_{2}$ 


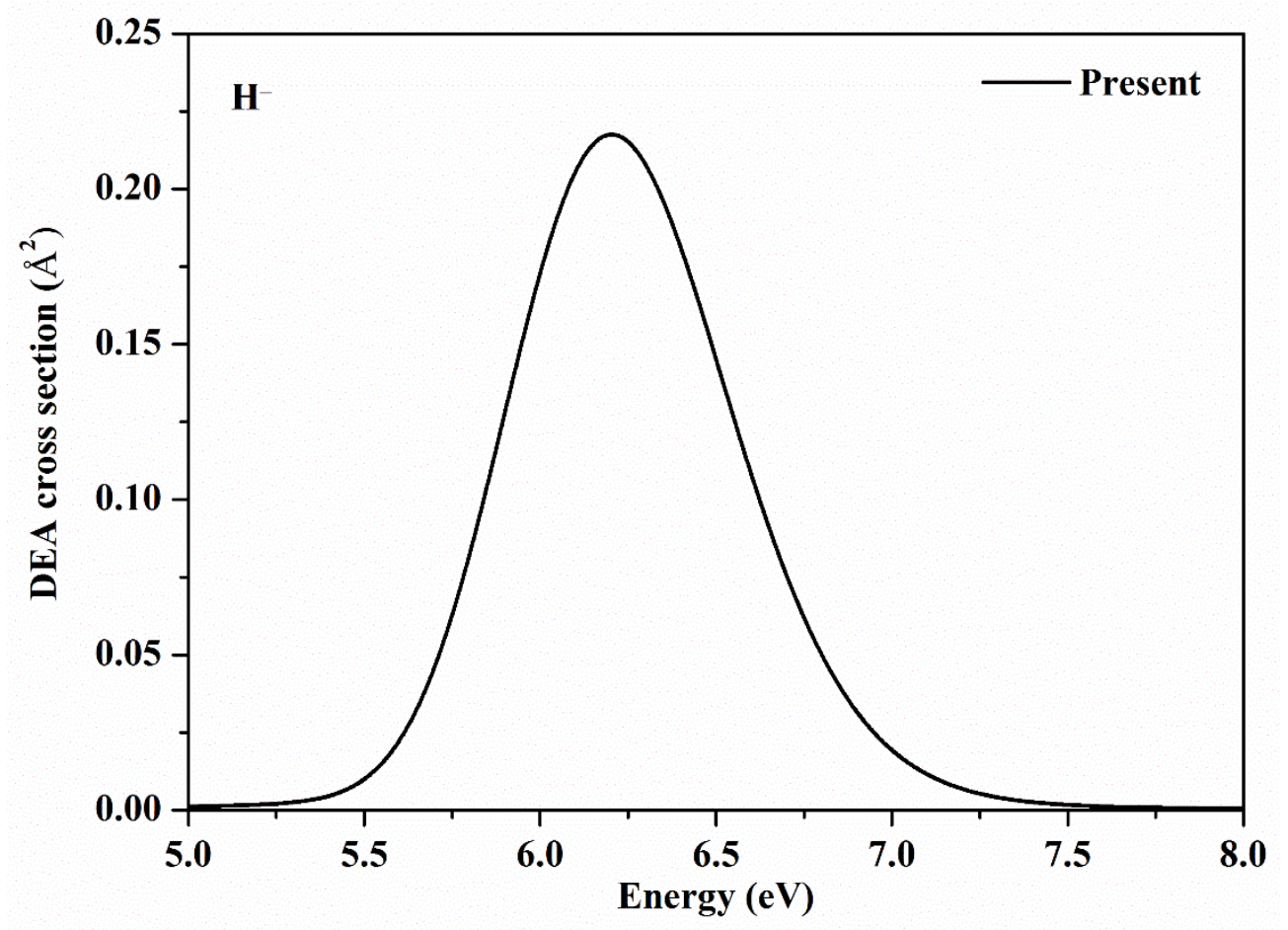

Figure 6. Dissociative electron attachment cross section for the formation of $\mathrm{H}^{-}$in $\mathrm{BeH}_{2}$. Solid line: Present.

Our predicted DEA cross section for the formation of the $\mathrm{H}^{-}$ion in $\mathrm{BeH}_{2}$ is plotted in figure 6 . The DEA cross section shows a peak at around $6.2 \mathrm{eV}$, and overlaps the threshold for the first excitation energy. This peak in the DEA cross section is due to the presence of the resonance detected in the present study at around $6.2 \mathrm{eV}$ as shown in Table 2. This is the first approximate theoretical prediction of the DEA cross section. The inputs used for this approximate prediction are the resonances detected in the present study and the neutral dissociation energy for the dissociation of $\mathrm{BeH}_{2}$ into the fragments $\mathrm{BeH}$ and $\mathrm{H}$ with the dissociation energy of $4.4 \mathrm{eV}$ [29]. The electron affinity of 0.704 is used for the hydrogen atom. The vibrational frequency of 1000 $\mathrm{cm}^{-1}$ is employed for the present calculation. The calculation is performed using the DEA estimator [30], which is part of the Quantemol-N system. Considering the importance of the DEA cross section for the plasma modelling applications, the semi-empirical predication such as this should be useful for use in modelling and comparisons.

\subsection{Effective collision frequency of electrons}

The two types of effective collision frequency, $\langle v\rangle$ and $\bar{v}^{-1}$ can be obtained from the momentum-transfer cross section for the electron-neutral interaction using the MaxwellBoltzmann distribution for the electrons as follows [31, 32]

$$
\langle v\rangle=\frac{8}{3 \pi^{\frac{1}{2}}} N\left(\frac{m_{e}}{2 K_{B} T_{e}}\right)^{\frac{5}{2}} \int_{0}^{\infty} v^{5} Q^{m}(v) e^{\frac{-m_{e} v^{2}}{2 K_{B} T_{e}}} d v
$$




$$
\bar{v}^{-1}=\frac{8}{3 \pi^{\frac{1}{2}} N}\left(\frac{m_{e}}{2 K_{B} T_{e}}\right)^{\frac{5}{2}} \int_{0}^{\infty} \frac{v^{3}}{Q^{m}(v)} e^{\frac{-m_{e} v^{2}}{2 K_{B} T_{e}}} d v
$$

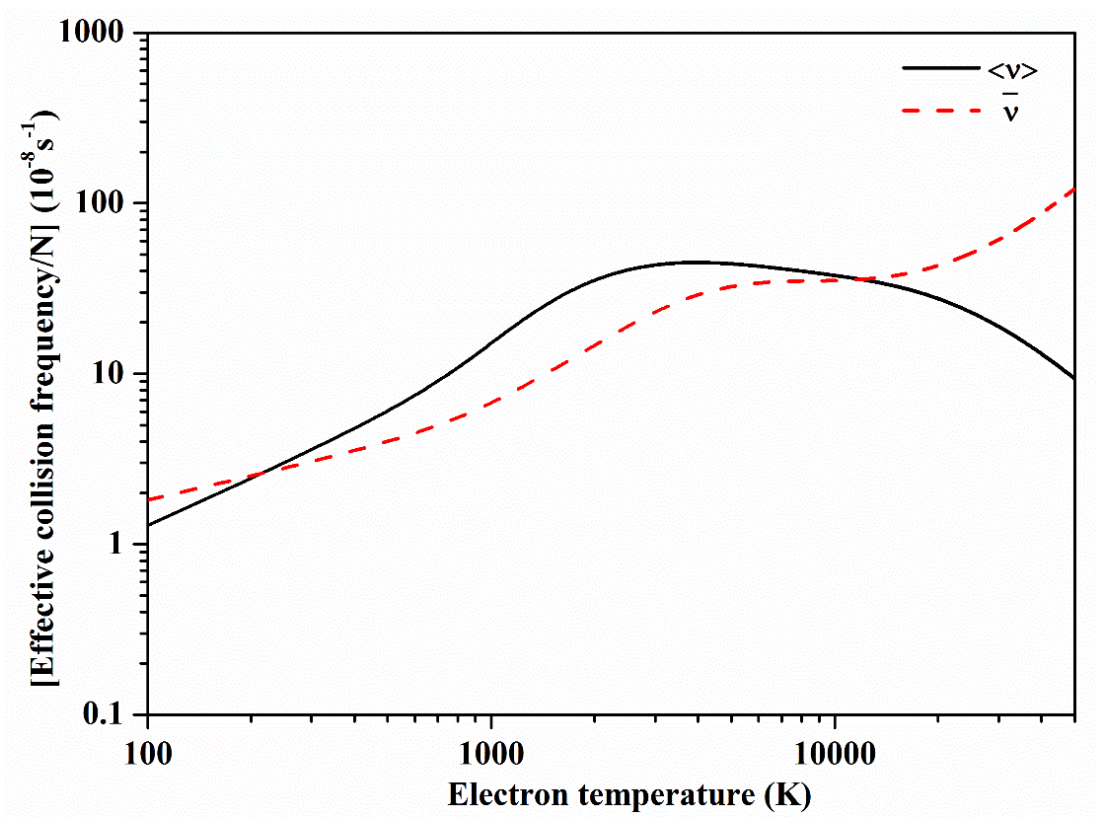

Figure 7. Effective collision frequency of the $\mathrm{BeH}_{2}$ molecule ground state as a function of electron temperature: Solid curve, $\langle v\rangle$; Dashed curve, $\bar{v}$.

Here, $N$ is the number density of molecules, $m_{e}$ is the electron mass, $K_{B}$ is the Boltzmann factor, $T_{e}$ is the electron temperature, $v$ is the velocity of the electron and $Q^{m}(v)$ is the velocity dependent MTCS. The collisional frequencies are plotted in Fig. 7 calculated using the MTCS data of $\mathrm{BeH}_{2}$ and are shown for the temperature range of 100 to $50000 \mathrm{~K}$. The collision frequencies could be easily employed to calculate transport properties like mean free path, mobility and diffusion coefficients and are important in the study of electron swarming through molecular gases.

\subsection{Mean free path, diffusivity and mobility of electrons}

The effective collision frequency as given in Eq. (4) is used for the calculations of transport properties such as mean free path, diffusivity and mobility [32] of electrons for a temperature range of 100 to $500000 \mathrm{~K}$ for a Maxwell-Boltzmann distribution.

The calculated temperature dependence of the electron mean free path, diffusion coefficient and mobility are shown in Figs. 8 (a)-8 (c) for a Maxwellian energy distribution. The mean free path, diffusion coefficient and mobility are calculated from the effective collision frequency by the expressions [32] given in Eq. (5),

$$
\lambda_{e}=\left(\frac{8 K_{B} T_{e}}{\pi m_{e}}\right)^{\frac{1}{2}} \bar{v}^{-1} ; D=\left(\frac{K_{B} T_{e}}{m_{e}}\right)^{\frac{1}{2}} \bar{v}^{-1} ; \mu=\left(\frac{e}{m_{e}}\right)^{\frac{1}{2}} \bar{v}^{-1}
$$




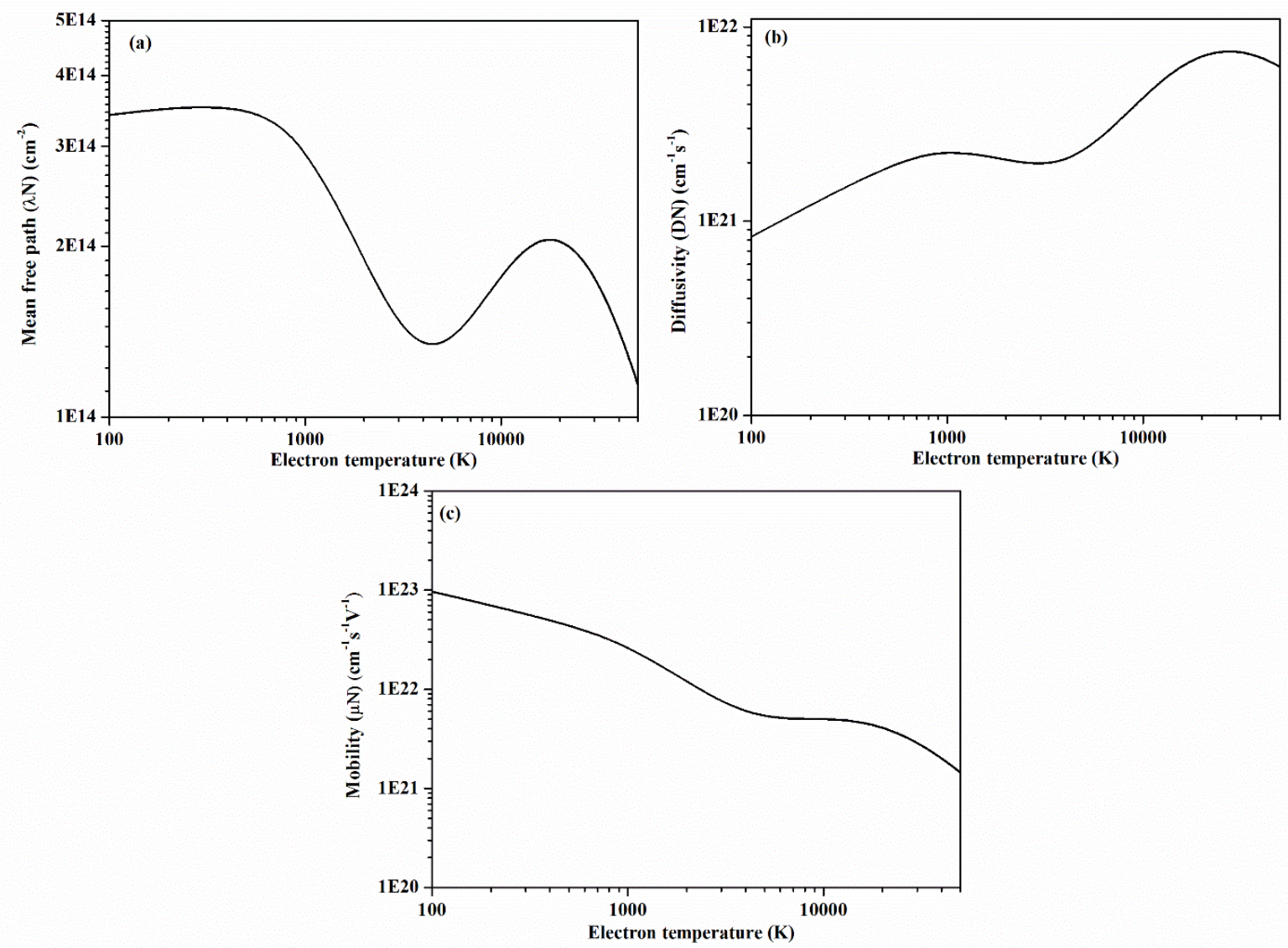

Figure 8. Electron temperature dependence of the (a) mean free path, $\lambda N\left(\mathrm{~cm}^{-2}\right)$ (b) diffusivity, $D N\left(\mathrm{~cm}^{-1} \mathrm{~s}^{-1}\right)$ and (c) mobility, $\mu N\left(\mathrm{~cm}^{-1} \mathrm{~s}^{-1} \mathrm{~V}^{-1}\right)$ for the $\mathrm{BeH}_{2}$ molecule.

There are no results in the literature to compare our data for these transport properties. The present results could be useful for the plasma community.

\section{Conclusion}

The elastic integral, differential, momentum transfer, dissociative electron attachment and electronic excitation cross-sections for low-energy electron collision with $\mathrm{BeH}_{2}$ molecule are reported using the $\mathrm{R}$-matrix method at $\mathrm{CC}$ approximations. We have performed the calculations using various SE, 1-state, 5-state, 10-state, 15-state, 20-state and 25-state CC models to check for the convergence of the results. Increasing the number of target states have substantial effect on the elastic and momentum transfer cross sections shifting the position of shape resonance to the lower energies and the results seems to converge for higher state calculations. A similar study investigating the effect of multichannel coupling on the cross sections of $\mathrm{CH}_{2}$ has been presented recently [33]. The vertical excitation energies with the 25-state $\mathrm{CC}$ model show excellent agreement with the EOM-CCSD calculations. This suggest that the uncertainty in the excitation cross section calculations are less than $10 \%$ if we compare the threshold of the vertical excitation energies for all the excited state. The DEA cross section for the formation of $\mathrm{H}^{-}$ion is also reported showing a peak at around $6.2 \mathrm{eV}$, associated with the narrow resonances detected in this energy region. The uncertainty in this cross section is likely to be 
larger, maybe as much as a factor of two, as we only use an estimator rather than a full nuclear motion treatment.

We also report collisional frequencies computed using the momentum transfer cross section within the approximation of a Maxwellian energy distribution function. The collision frequency is used to calculate some transport properties such mean free path, diffusivity and mobility of the electrons using a Maxwell-Boltzmann distribution. The cross section data and transport properties reported in this article are important for fusion plasma applications.

\section{Supplementary material}

The numerical value of all the cross sections, collision frequencies and transport properties are provided in a supplementary file.

\section{Acknowledgement}

D.G. is pleased to acknowledge the support of this research to the National Fusion Research Institute (NFRI), Korea for the research grant and scholarship.

\section{References}

[1] Bartschat K and Kushner M J 2016 Proc. Nat. Acad. Sci. 1137026.

[2] Federici G 2006 Phys. Scr. T124 1.

[3] R. Doerner, M.J. Baldwin, D. Buchenauer, G. De Temmerman and D. Nishijima 2009 J. Nucl. Mater. 390681.

[4] Björkas C, Vörtler K, Nordlund K, Nishijima D and Doerner R 2009 New J. Phys. 11 123017.

[5] Celiberto R, Baluja K L and Janev R K 2013 Plasma Sources Sci. Technol. 22015008.

[6] Duxbury G, Stamp M F and Summers H P 1998 Plasma Phys. Controlled fusion 40 361.

[7] Darby-Lewis D, Tennyson J, Lawson K D, Yurchenko S N, Stamp M F, Shaw A, Brezinsek S and JET Contributors 2018 J. Phys. B: At. Mol. Opt. Phys. (in press).

[8] Maihom T, Sukuba I, Janev R, Becker K, Märk T, Kaiser A, Limtrakul J, Urban J, Mach P and Probst M 2013 Eur. Phys. J. D 675.

[9] Darby-Lewis D, Masin Z and Tennyson J 2017 J. Phys. B: At. Mol. Opt. Phys. 50 175201.

[10] https://cccbdb.nist.gov/

[11] Frisch M J et al. 2013 Gaussian 09 Revision D.01 Gaussian, Inc., Wallingford CT.

[12] Burke P G 2011 R-matrix Theory of Atomic Collision (Springer Series on Atomic, Optical, and Plasma Physics, 2011)

[13] Tennyson J 2010 Phys. Rep. 49129.

[14] Takatsuka K and McKoy V 1981 Phys. Rev. A 242473.

[15] Schneider B I and Rescigno T N 1988 Phys. Rev. A 373749.

[16] Carr J M, Galiatsatos P G, Gorfinkiel J D, Harvey A G, Lysaght M A, Madden D, Mašín Z, Plummer M, Tennyson J and Varambhia H N 2012 Eur. Phys. J. D 6658.

[17] Tennyson J, Brown D B, Munro J, Rozum I, Varambhia H N and Vinci N $2007 \mathrm{~J}$. 
Phys.: Conf. Series 86012001.

[18] Arthurs A M and Dalgarno A 1960 Proc. Phys. Soc., London, Sect. A 256540.

[19] Tennyson J 1996 J. Phys B: At. Mol. Opt. Phys. 291817.

[20] Zatsarinny O 2006 Comput. Phys. Commun. 174273

[21] Gedeon V, Gedeon S, Lazur V, Nagy E, Zatsarinny O and Bartschat K 2018 J. Phys. B: At. Mol. Opt. Phys. 51035004

[22] Gedeon V, Gedeon S, Lazur V, Nagy E, Zatsarinny O and Bartschat K 2015 Phys. Rev. A 92052701.

[23] Zatsarinny O, Bartschat K, Gedeon S, Gedeon V, Lazur V 2006 Phys. Rev. A 74 052708

[24] Faure A, Gorfinkiel J D, Morgan L A and Tennyson J 2002 Comput. Phys. Commun. 144224.

[25] Gailitis M 1976 J. Phys. B 9843.

[26] Sanna N and Gianturco F A 1998 Comput. Phys. Commun. 114142.

[27] Tennyson J and Noble C 1984 J Comput. Phys. Commun. 33421.

[28] Breit G and Wigner E 1936 Phys. Rev. 49519.

[29] Price W C, Passmore T R and Roessler D M 1963 Discuss. Faraday Soc. 35201.

[30] Munro J J, Harrison S, Fujimoto M M and Tennyson J 2012 J. Phys.: Conf. Series 388 012013.

[31] Itikawa Y 1973 Phys. Fluids 16831.

[32] Baille P, Chang J S, Claude A, Hobson R M, Ogram G L, Yau A W 1981 J. Phys B: At. Mol. Phys. 141485.

[33] Wang K, Zhang H, Huang X, Liu Yufang and Sun J, Phys. Rev. A 97, 012703 (2018) 